\title{
DEGREE AND SOBOLEV SPACES
}

\author{
Hä̈m Brezis ${ }^{(1)(2)}$, YAnyan Li ${ }^{(2)}$, Petru Mironescu $^{(3)}$ And Louis NiRenberg ${ }^{(4)}$ \\ Dedicated to Jurgen Moser in friendship and admiration
}

\section{Introduction.}

J. Rubinstein and P. Sternberg established in [9] the following result. Let $\Omega$ be a solid 3-dimensional torus, i.e., $\Omega=S^{1} \times \Lambda$ where $\Lambda$ is the unit disc in $\mathbb{R}^{2}$. Let $u \in H^{1}\left(\Omega ; S^{1}\right)$. For a.e. $\lambda \in \Lambda$ the map

$$
x \in S^{1} \mapsto u(x, \lambda) \in S^{1}
$$

belongs to $H^{1}\left(S^{1}, S^{1}\right)$; thus it is continuous and has a degree. Conclusion:

$$
\operatorname{deg}(u(\cdot, \lambda)) \text { is independent of } \lambda \text {. }
$$

This result is somewhat surprising because $H^{1}$ functions in 3-d need not be continuous, and not even in VMO. If $\Omega$ were a 2 -d annulus, $\Omega=S^{1} \times(0,1)$, instead of a 3 -d torus the conclusion would still be surprising; however, in this case one can give a straightforward proof via the $H^{1 / 2}\left(S^{1}, S^{1}\right)$ degree theory of L. Boutet de Monvel and O. Gabber (see [4] and also [5]). Indeed, by standard trace theory, the map

$$
\lambda \in(0,1) \mapsto u(\cdot, \lambda) \in H^{1 / 2}\left(S^{1}, S^{1}\right)
$$

is continuous. We recall that any map $\varphi \in H^{1 / 2}\left(S^{1}, S^{1}\right)$ has a degree which depends continuously on the $H^{1 / 2}$ norm. Therefore $\operatorname{deg}(u(\cdot, \lambda))$ is well-defined for every $\lambda \in(0,1)$ and is independent of $\lambda$.

By contrast, in 3-d, there is no similar argument since a general $H^{1}$ function does not have trace on every line.

In this paper we first give, in Section 1, a direct generalization with simple proof. We then present in Section 2 a still more general result which holds in fractional Sobolev spaces.

Acknowledgments: The first author (H.B.) is partially supported by a European Grant ERB FMRX CT98 0201. The second author (Y.L.) is partially supported by NSF Grant DMS-9706887 and a Rutgers University Research Council grant. Part of this work was done when the third author (P.M.) was visiting Rutgers University; he thanks the Mathematics Department for its invitation and hospitality. The visit of the fourth author (L.N.) to Paris was supported by the Institut Universitaire de France and he thanks it for its hospitality. 


\section{Section 1.}

Let $X$ and $Y$ be compact, oriented, $n$-dimensional smooth manifolds without boundary, $Y$ is connected. Let $\Lambda$ be a domain in $\mathbb{R}^{k}$. Let $u$ be a map from $\Omega=X \times \Lambda$ into $Y$ which belongs to $W^{1, n+1}(\Omega, Y)$, i.e., if $Y$ is smoothly embedded in some $\mathbb{R}^{N}$ then $u$ is a map from $\Omega$ into $\mathbb{R}^{N}$ having each component in $W^{1, n+1}$ and such that $u(x, \lambda) \in Y$ a.e. in $\Omega$. For a.e. $\lambda \in \Lambda, u(\cdot, \lambda)$ belongs to $W^{1, n+1}(X, Y)$; so it is continuous and therefore $\operatorname{deg}(u(\cdot, \lambda))$ is well defined.

Theorem 1. $\operatorname{deg}(u(\cdot, \lambda))$ is independent of $\lambda$ and we call it simply deg $u$. Moreover, deg $u$ is stable under convergence in $W^{1, n}(\Omega)$, i.e., if a sequence $\left(u_{j}\right)$ in $W^{1, n+1}(\Omega)$ converges in the $W^{1, n}$ norm to some $u \in W^{1, n+1}(\Omega)$, then deg $u_{j}=\operatorname{deg} u$ for sufficiently large $j$.

Remark 1. The result need not hold if $u$ is merely in $W^{1, p}(\Omega, Y)$ with $p<n+1$. Here is an example. Let $X=Y=S^{n}$ and let $\Omega=(0,2)^{k}$

$$
u(x, \lambda)=\frac{x-\lambda_{1} e_{1}}{\left|x-\lambda_{1} e_{1}\right|}
$$

It is easily seen that $u \in W^{1, p}(\Omega, Y)$ for every $p<n+1$. On the other hand $\operatorname{deg}(u(\cdot, \lambda))=0$ for $\lambda_{1}>1$ and $\operatorname{deg}(u(\cdot, \lambda))=1$ for $\lambda_{1}<1$.

The proof of the theorem uses a standard representation of the degree of a $C^{1} \operatorname{map} \varphi$ as an integral. If $\omega$ is a smooth $n$-form on $Y$ with

$$
\int_{Y} \omega=1
$$

then

$$
\operatorname{deg} \varphi=\int_{X} \omega \circ \varphi
$$

where $\omega \circ \varphi$ is the pull-back of $\omega$ by $\varphi$ (see e.g. [8]). That formula still holds (by density) if $\varphi \in C^{0} \cap W^{1, n}$. (In fact, it suffices that $\varphi \in W^{1, n}$ since it is then in VMO and VMO-maps have a degree, see [5]).

Proof of Theorem 1. It is convenient to work with a special form $\omega$ on $Y$ having small support. For then we can use one fixed local coordinate system near a point. Assume $0 \in Y \subset \mathbb{R}^{N}$; we may also choose the embedding $e$ of $Y$ into $\mathbb{R}^{N}$ in such a way that, in a neighborhood $V$ of 0 in $\mathbb{R}^{N}$,

$$
e(Y)=\left\{y ; \quad y^{n+1}=y^{n+2}=y^{N}=0\right\} .
$$

Let $\zeta$ be a smooth function with support in $V$ such that

$$
\int \zeta\left(y^{1}, y^{2}, \ldots y^{n}, 0, \ldots 0\right) d y^{1} \ldots d y^{n}=1
$$


We consider the $n$-form $\tilde{\omega}$ on $\mathbb{R}^{N}$,

$$
\tilde{\omega}=\zeta\left(y^{1}, y^{2}, \ldots, y^{N}\right) d y^{1} \wedge \cdots \wedge d y^{n}
$$

and take as $w$ on $Y$, the pull back of $\tilde{\omega}$ under $e$; in our local coordinates it has the form

$$
\omega=\zeta\left(y^{1}, y^{2}, \ldots y^{n}, 0, \ldots, 0\right) d y^{1} \wedge \cdots \wedge d y^{n}
$$

and thus $\int_{Y} \omega=1$.

We have to prove that

$$
\int_{X} \omega \circ u(\cdot, \lambda)
$$

is independent of $\lambda$, a.e. in $\Lambda$. The natural argument would be to differentiate the integral with respect to the parameter $\lambda$. However, the integrand in (1) already involves first order derivatives of $u$ and $\lambda$-differentiation introduces second-order derivatives. We get rid of these by integration by parts. To carry this out we use approximation by smooth functions.

Let $u_{\varepsilon}$ be a family of smooth maps from $\bar{\Omega}$ into $\mathbb{R}^{N}$ converging, as $\varepsilon \rightarrow 0$, to $u$ in $W^{1, n+1}$. Note that, in general, the $u_{\varepsilon}$ 's do not map into $Y$ (and not even into a neighborhood of $Y$, see [2]). Set

$$
\psi_{\varepsilon}(\lambda)=\int_{X} \tilde{\omega} \circ u_{\varepsilon}(\cdot, \lambda)
$$

and differentiate $\psi_{\varepsilon}$ with respect to one of the $\lambda^{\prime}$ 's, still denoted by $\lambda$.

We find, with $u_{\varepsilon \lambda}^{i}=\frac{\partial}{\partial \lambda} u_{\varepsilon}^{i}$,

$$
\begin{aligned}
\frac{\partial}{\partial \lambda} \psi_{\varepsilon}(\lambda) & =\int_{X} \sum_{1}^{N} \frac{\partial \zeta}{\partial y^{j}}\left(u_{\varepsilon}\right) u_{\varepsilon \lambda}^{j} d u_{\varepsilon}^{1} \wedge \ldots \wedge d u_{\varepsilon}^{n} \\
& +\int_{X} \zeta\left(u_{\varepsilon}\right) d u_{\varepsilon \lambda}^{1} \wedge d u_{\varepsilon}^{2} \wedge \ldots \wedge d u_{\varepsilon}^{n} \\
& +\cdots+\int_{X} \zeta\left(u_{\varepsilon}\right) d u_{\varepsilon}^{1} \wedge \cdots \wedge d u_{\varepsilon}^{n-1} \wedge d u_{\varepsilon \lambda}^{n} .
\end{aligned}
$$

Now

$$
\begin{aligned}
\int_{X} \zeta\left(u_{\varepsilon}\right) d u_{\varepsilon \lambda}^{1} \wedge d u_{\varepsilon}^{2} \wedge \cdots \wedge d u_{\varepsilon}^{n} & =\int_{X} d\left[\zeta\left(u_{\varepsilon}\right) u_{\varepsilon \lambda}^{1} d u_{\varepsilon}^{2} \wedge \cdots \wedge d u_{\varepsilon}^{n}\right] \\
& -\int_{X} \sum_{1}^{N} u_{\varepsilon \lambda}^{1} \frac{\partial \zeta}{\partial y^{j}}\left(u_{\varepsilon}\right) d u_{\varepsilon}^{j} \wedge d u_{\varepsilon}^{2} \wedge \cdots \wedge d u_{\varepsilon}^{n} \\
& =-\int_{X} u_{\varepsilon \lambda}^{1} \frac{\partial \zeta}{\partial y^{1}}\left(u_{\varepsilon}\right) d u_{\varepsilon}^{1} \wedge d u_{\varepsilon}^{2} \wedge \cdots \wedge d u_{\varepsilon}^{n} \\
& -\int_{X} \sum_{n+1}^{N} u_{\varepsilon \lambda}^{1} \frac{\partial \zeta}{\partial y^{j}}\left(u_{\varepsilon}\right) d u_{\varepsilon}^{j} \wedge d u_{\varepsilon}^{2} \wedge \cdots \wedge d u_{\varepsilon}^{n}
\end{aligned}
$$


Similar expressions hold for the term after this one in (2). Inserting these expressions into (2), we find

$$
\begin{aligned}
& \frac{\partial}{\partial \lambda} \psi_{\varepsilon}(\lambda)=\int_{X} \sum_{n+1}^{N} \frac{\partial \zeta}{\partial y^{j}}\left(u_{\varepsilon}\right) u_{\varepsilon \lambda}^{j} d u_{\varepsilon}^{1} \wedge \cdots \wedge d u_{\varepsilon}^{n} \\
& \quad-\int_{X} \sum_{n+1}^{N} \frac{\partial \zeta}{\partial y^{j}}\left(u_{\varepsilon}\right)\left[u_{\varepsilon \lambda}^{1} d u_{\varepsilon}^{j} \wedge d u_{\varepsilon}^{2} \wedge \cdots \wedge d u_{\varepsilon}^{n}\right. \\
& \left.\quad+u_{\varepsilon \lambda}^{2} d u_{\varepsilon}^{1} \wedge d u_{\varepsilon}^{j} \wedge d u_{\varepsilon}^{3} \wedge \cdots \wedge d u_{\varepsilon}^{n}+\cdots+u_{\varepsilon \lambda}^{n} d u_{\varepsilon}^{1} \wedge \cdots \wedge d u_{\varepsilon}^{n-1} \wedge d u_{\varepsilon}^{j}\right] .
\end{aligned}
$$

Next we claim that, as $\varepsilon \rightarrow 0$,

$$
\int_{\Omega}\left|\frac{\partial \psi_{\varepsilon}}{\partial \lambda}\right| \rightarrow 0
$$

Indeed by (3) we have

$$
\left|\frac{\partial \psi_{\varepsilon(\lambda)}}{\partial \lambda}\right| \leq C \sum_{n+1}^{N} \int_{X}\left|\frac{\partial \zeta}{\partial y^{j}}\left(u_{\varepsilon}\right)\right|\left|D u_{\varepsilon}^{j}\right|\left|D u_{\varepsilon}\right|^{n}
$$

where $D$ denotes the full gradient (in $x$ and $\lambda$ ).

Thus

$$
\int_{\Lambda}\left|\frac{\partial \psi_{\varepsilon}}{\partial \lambda}\right| \leq C \sum_{n+1}^{N}\left[\int_{\Omega}\left|\frac{\partial \zeta}{\partial y^{j}}\left(u_{\varepsilon}\right)\right|^{n+1}\left|D u_{\varepsilon}^{j}\right|^{n+1}\right]^{1 /(n+1)}\left\|u_{\varepsilon}\right\|_{W^{1, n+1}}^{n}(\Omega) .
$$

Since $u_{\varepsilon} \rightarrow u$ in $W^{1, n+1}(\Omega)$ we have

$$
\int_{\Lambda}\left|\frac{\partial \psi_{\varepsilon}}{\partial \lambda}\right| \leq C \sum_{n+1}^{N}\left[\int_{\Omega}\left|\frac{\partial \zeta}{\partial y^{j}}\left(u_{\varepsilon}\right)\right|^{n+1}\left|D u_{\varepsilon}^{j}\right|^{n+1}\right]^{1 /(n+1)}
$$

Next observe that (passing to a subsequence)

$$
\left|\frac{\partial \zeta}{\partial y^{j}}\left(u_{\varepsilon}\right)\right|\left|D u_{\varepsilon}^{j}\right| \rightarrow\left|\frac{\partial \zeta}{\partial y^{j}}(u)\right|\left|D u^{j}\right| \quad \text { a.e. on } \Omega
$$

and, for $j>n, \frac{\partial \zeta}{\partial y^{j}}(u) D u^{j}=0$ a.e. on $\Omega$ (since on the set $\{(x, \lambda) ; u(x, \lambda) \in V\}, u^{j}=0$ for $j=n+1, \ldots, N$ and hence $D u^{j}=0$ ). On the other hand (passing to a subsequence) we 
may assume that $\left|D u_{\varepsilon}^{j}\right|$ is bounded by a fixed function in $L^{n+1}(\Omega)$ and hence, by dominated convergence,

$$
\int_{\Omega}\left|\frac{\partial \zeta}{\partial y^{j}}\left(u_{\varepsilon}\right)\right|^{n+1}\left|D u_{\varepsilon}^{j}\right|^{n+1} \rightarrow 0, \quad \text { for } j>n
$$

which yields (4).

Finally we claim that

$$
\psi_{\varepsilon}(\lambda) \rightarrow \psi(\lambda)=\int_{X} \tilde{\omega} \circ u(\cdot, \lambda)=\int_{X} \omega \circ u(\cdot, \lambda) \quad \text { in } L^{(n+1) / n}(\Lambda)
$$

Indeed the integrand in $\psi_{\varepsilon}$ can be estimated pointwise by

$$
\left|\tilde{\omega} \circ u_{\varepsilon}\right| \leq C\left|D u_{\varepsilon}\right|^{n}
$$

and thus (passing to a subsequence)

$$
\left|\tilde{\omega} \circ u_{\varepsilon}-\tilde{\omega} \circ u\right| \leq f
$$

where $f$ is a fixed function in $L^{(n+1) / n}(\Omega)$.

Therefore

$$
\left|\psi_{\varepsilon}(\lambda)-\psi(\lambda)\right|^{(n+1) / n} \leq C \int_{X}|f(x, \lambda)|^{(n+1) / n} d x
$$

and the right-hand side is a fixed function in $L^{1}(\Lambda)$. The claim (5) follows, again by dominated convergence, since $\psi_{\varepsilon}(\lambda) \rightarrow \psi(\lambda)$ a.e.

Combining (4) and (5) we see that $\psi \in W^{1,1}(\Lambda)$ and

$$
\frac{\partial \psi}{\partial \lambda}=0
$$

Hence $\psi$ is independent of $\lambda$.

The last assertion in the theorem, i.e., stability of degree under $W^{1, n}$ convergence follows easily from the formula

$$
\operatorname{deg} u=\oint_{\Lambda} \int_{X} \omega \circ u
$$

and the fact that the integrand in the right-hand side involves $n$-products of derivatives of $u$.

Remark 2. The above computation for computing the $\lambda$-derivative of a pull back can be expressed globally, and more succinctly, in terms of differential forms. 
Namely, consider $X$ and $\Lambda$ as above and a smooth map $u$ from $\Omega=X \times \Lambda$ into an oriented manifold $Z$ (in the case above, $Z=\mathbb{R}^{N}$ ). Let $\tilde{\omega}$ be a smooth $n$-form on $Z$. The $\lambda$-derivative of the pullback $\tilde{\omega} \circ u(\cdot, \lambda)$ is simply

$$
\partial_{\lambda} \tilde{\omega} \circ u(\cdot, \lambda)=d A+B
$$

where $A$ and $B$ are $(n-1)$ and $n$-forms respectively on $X$. They are expressed using the tangent vector $u_{\lambda}$ which is defined at points of $Z$ in the image of $u(\cdot, \lambda) . A$ and $B$ are given by

$$
\begin{gathered}
\left.A=(\tilde{\omega}\lrcorner u_{\lambda}\right) \circ u \\
\left.B=(d \tilde{\omega}\lrcorner u_{\lambda}\right) \circ u .
\end{gathered}
$$

Here $u_{\lambda}$ is the $\lambda$-derivative (say with respect to one of the $\lambda$ coordinates) of $u$. The symbol \lrcorner denotes contraction of a differential form and a vector (see [7]).

Formula (6) holds for a smooth map. It still holds for maps in $W^{1, n+1}$, provided one interprets (6) in the distribution sense. In fact, the coefficients of $\tilde{\omega} \circ u$ and of $A$ are $n$ products of functions in $L^{n+1}$, the coefficients of $B$ are $n+1$ products of functions in $L^{n+1}$. To justify (6) in such generality one smoothes $u$ by $u_{\varepsilon}$ as above, mapping however into a high dimension Euclidean space in which $Z$ is embedded.

In the special case that $\operatorname{dim} Z=\operatorname{dim} X$ (for example if $Z=Y$ as above) then $d \tilde{\omega}=0$ and thus $B=0$.

Warning: The reader might think that in this case (6) holds with $B=0$ assuming only that $u \in W^{1, n}$. This is not true as the counterexample in Remark 1 shows.

When using degree theory one often considers $X$, the domain space with a boundary, $Y$ connected and open. One wishes to compute the degree of $u: X \rightarrow Y$ at some point $y \in Y$ which is not in the image of the boundary (if the map $u \in C(\bar{X})$, Theorem 1 easily extends to such a situation). Here is one form of such a result:

Let $X$ be an open subset of an $n$-dimensional smooth oriented manifold $\tilde{X}$ with $\bar{X}$, the closure of $X$, compact in $\widetilde{X}$ and $\partial X$ smooth. Let $Y$ be an open oriented, connected, $n$-dimensional smooth Riemannian manifold. Let $\Lambda$ be a domain in $\mathbb{R}^{k}$. Let $u$ be a map from $\Omega=X \times \Lambda$ into $Y$ which belongs to $W^{1, n+1}(\Omega, Y)$. For a.e. $\lambda \in \Lambda, u(\cdot, \lambda)$ belongs to $W^{1, n+1}(X, Y)$ so it is continuous in $\bar{X}$. Assume that $y \in Y$ is such that for some $\delta>0$ and for a.e. $\lambda$ as above,

$$
\operatorname{dist}(y, u(\partial X, \lambda)) \geq \delta
$$

Then the degree of $u$ at $y, \operatorname{deg}(u(\cdot, \lambda), X, y)$ is well defined.

Theorem $\mathbf{1}^{\prime} \cdot \operatorname{deg}(u(\cdot, \lambda), X, y)$ is independent of $\lambda$.

The proof is just the same as that of Theorem 1 . We may suppose that $y$ is the origin in $\mathbb{R}^{N}$ and that $Y$ near 0 is flat. Then we take the forms $\tilde{\omega}$ and $w$ as above, with supp $\omega$ lying in a $\delta / 2$ neighborhood (with respect to the metric on $Y$ ) of $y$. Then proceed as before. 


\section{Section 2.}

Let $X, Y$ and $\Lambda$ be as in Theorem 1 and let $u$ be a map from $\Omega=X \times \Lambda$ into $Y$ which belongs to $W^{s, p}(\Omega, Y)$ with $s>0$ and $1<p<\infty$. Recall that for a.e. $\lambda \in \Lambda, u(\cdot, \lambda)$ belongs to $W^{s, p}(X, Y)$. This is clear if $s$ is an integer; when $0<s<1$ such property is an easy consequence of the equivalence of two $W^{s, p}$ norms in $\mathbb{R}^{m}$ :

$$
\|f\|_{W^{s, p}}^{p}=\|f\|_{L^{p}}^{p}+\int_{\mathbb{R}^{m}} \int_{\mathbb{R}^{m}} \frac{|f(x)-f(y)|^{p}}{|x-y|^{m+s p}} d x d y
$$

and

$$
\|\mid f\|_{W^{s, p}}^{p}=\|f\|_{L^{p}}^{p}+\sum_{i=1}^{m} \int_{0}^{1} \int_{\mathbb{R}^{m}} \frac{\left|f\left(x+t e_{i}\right)-f(x)\right|^{p}}{t^{s p}} d x d t
$$

(see e.g. Adams [1], p. 208-214 or Triebel [10]). The case of a general $s>0$ follows easily.

Assuming further that

$$
s p \geq n+1
$$

we find that for a.e. $\lambda \in \Lambda, u(\cdot, \lambda) \in W^{s, p}(X, Y) \subset C^{0}(X, Y)$; therefore $\operatorname{deg}(u(\cdot, \lambda))$ is well defined for a.e. $\lambda \in \Lambda$.

Theorem 2. Assume that $u \in W^{s, p}(\Omega, Y)$ and that (9) holds. Then

$$
\operatorname{deg}(u(\cdot, \lambda)) \quad \text { is independent of } \lambda \text {. }
$$

Moreover, this degree is stable under convergence in any $W^{s^{\prime}, p^{\prime}}$ norm provided $s^{\prime} p^{\prime} \geq n$.

Proof. We may assume that $0<s \leq 1$ and the case $s>1$ is handled with minor modifications. In this generality there is no integral representation for the degree and the argument is quite different from the proof of Theorem 1. Clearly it suffices to prove that the degree is locally constant a.e. Hence it suffices to consider the case where $\Lambda=(0,1)^{k}$. We assume first that $k=1$ and the general case will be done by reduction to $k=1$ as in Bethuel and Demengel [3] (Lemma A.1).

Case where $\boldsymbol{\Lambda}=(\mathbf{0}, \mathbf{1})$. By the standard trace theory a map $u \in W^{s, p}(X \times(0,1), Y)$ can be identified with a map $u \in C\left([0,1], W^{s-1 / p, p}(X, Y)\right.$. Since $(s-1 / p) p=s p-1 \geq n$, $W^{s-1 / p, p}(X) \subset \operatorname{VMO}(X)$ (see e.g. [5]); we also recall that there is a degree theory on $\operatorname{VMO}(X, Y)$ and that this degree is stable under small VMO perturbation. In this case $\operatorname{deg}(u(\cdot, \lambda))$ is well defined for every $\lambda \in[0,1]$ and it is independent of $\lambda$. 
Case where $\boldsymbol{\Lambda}=(\mathbf{0}, \mathbf{1})^{\mathbf{k}}$. We start with two lemmas:

Lemma 1. The map $\lambda \mapsto \operatorname{deg}(u(\cdot, \lambda))=\psi(\lambda)$ is measurable.

Proof. Consider a sequence $\left(u_{j}\right)$ of smooth functions on $X \times \bar{\Lambda} \rightarrow \mathbb{R}^{N}$ ( $Y$ is embedded in $\mathbb{R}^{N}$ ) such that

$$
u_{j} \rightarrow u \quad \text { in } W^{s, p}(X \times \bar{\Lambda})
$$

Passing to a subsequence (and using the equivalence of norms mentioned above) we may assume that for a.e. $\lambda \in \Lambda$

$$
u_{j}(\cdot, \lambda) \rightarrow u(\cdot, \lambda) \quad \text { in } W^{s, p}(X)
$$

In particular for a.e. $\lambda \in \Lambda$,

$$
u_{j}(\cdot, \lambda) \rightarrow u(\cdot, \lambda) \quad \text { uniformly in } X .
$$

Let $\delta>0$ be sufficiently small so that in the closed $\delta$-neighborhood $N_{\delta}(Y)$ of $Y$ in $\mathbb{R}^{N}$ the projection $P_{Y}$ onto $Y$ is well defined.

For every $j=1,2, \ldots$ and every $\lambda \in \bar{\Lambda}$ set

$$
\gamma_{j}(\lambda)=\operatorname{Sup}_{x \in X} \operatorname{dist}\left(u_{j}(x, \lambda), Y\right)
$$

(so that each $\gamma_{j}$ is continuous-even Lipschitz-in $\lambda$ ) and

$$
\psi_{j}(\lambda)= \begin{cases}\operatorname{deg}\left(P_{Y}\left(u_{j}(\cdot, \lambda)\right) \frac{\left(\delta-\gamma_{j}(\lambda)\right)}{\delta}\right. & \text { if } \gamma_{j}(\lambda) \leq \delta \\ 0 & \text { if } \gamma_{j}(\lambda)>\delta\end{cases}
$$

In view of (10) it is clear that

$$
\psi_{j}(\lambda) \rightarrow \psi(\lambda), \quad \text { as } j \rightarrow \infty \text {, a.e. in } \lambda \in \Lambda \text {. }
$$

On the other hand, it is easy to check that for every $j$, the function $\lambda \mapsto \psi_{j}(\lambda)$ is continuous on $\bar{\Lambda}$. Thus $\psi$ is measurable on $\Lambda$.

The second lemma is purely measure theoretical.

Lemma 2. Let $\Lambda=(0,1)^{k}$ and let $\psi$ be a measurable function on $\Lambda$ such that for each $1 \leq i \leq n$ and for a.e. $\left(\lambda_{1}, \ldots \lambda_{i-1}, \lambda_{i+1}, \ldots \lambda_{k}\right)$ in $(0,1)^{k-1}$, the function

$$
a \in(0,1) \mapsto \psi\left(\lambda_{1}, \ldots \lambda_{i-1}, a, \lambda_{i+1}, \ldots \lambda_{k}\right)
$$

is constant a.e. on $(0,1)$. Then $\psi$ is constant a.e. on $\Lambda$.

Proof. We may always assume that $\psi$ is also bounded (and thus integrable) since otherwise we may replace $\psi$ by Arctan $\psi$. By the triangle inequality, with

$$
\lambda=\left(\lambda_{1}, \ldots \lambda_{k}\right) \text { and } \mu=\left(\mu_{1}, \ldots \mu_{k}\right)
$$


we have

$$
\begin{aligned}
\mid \psi(\lambda) & -\psi(\mu)|\leq| \psi\left(\lambda_{1}, \lambda_{2}, \ldots \lambda_{k-1}, \lambda_{k}\right)-\psi\left(\lambda_{1}, \lambda_{2}, \ldots \lambda_{k-1}, \mu_{k}\right) \mid \\
& +\left|\psi\left(\lambda_{1}, \lambda_{2}, \ldots \lambda_{k-1}, \mu_{k}\right)-\psi\left(\lambda_{1}, \lambda_{2}, \ldots \mu_{k-1}, \mu_{k}\right)\right| \\
& +\cdots+\left|\psi\left(\lambda_{1}, \mu_{2}, \ldots \mu_{k-1}, \mu_{k}\right)-\psi\left(\mu_{1}, \mu_{2}, \ldots \mu_{k-1}, \mu_{k}\right)\right|
\end{aligned}
$$

It follows from the assumption that

$$
\int_{(0,1)^{k}} \int_{(0,1)^{k}}|\psi(\lambda)-\psi(\mu)| d \lambda d \mu=0 .
$$

Consequently, $\psi(\lambda)-\psi(\mu)=0$ a.e. on $(0,1)^{k} \times(0,1)^{k}$ which implies that $\psi(\lambda)$ is constant a.e. on $(0,1)^{k}$.

We now return to the proof of the theorem and, in view of the $\Lambda=(0,1)$ case, apply Lemma 2 to $\psi(\lambda)=\operatorname{deg}(u(\cdot, \lambda))$ to conclude that $\operatorname{deg}(u(\cdot, \lambda))$ is constant a.e. in $(0,1)^{k}$.

To establish the stability under $W^{s^{\prime}, p^{\prime}}$ convergence with $s^{\prime} p^{\prime} \geq n$ we argue as follows. Consider a sequence $\left(u_{j}\right)$ in $W^{s, p}$ converging in the $W^{s^{\prime}, p^{\prime}}$ norm to some $u \in W^{s, p}$ with $s p \geq n+1$. As in Lemma 1, passing to a subsequence we may assume that, for a.e. $\lambda \in \Lambda$,

$$
u_{j}(\cdot, \lambda) \rightarrow u(\cdot, \lambda) \quad \text { in } W^{s^{\prime}, p^{\prime}}(X) .
$$

Since $s^{\prime} p^{\prime} \geq n, W^{s^{\prime}, p^{\prime}}$ is contained in VMO, and we may infer from the result of [5] that, for a.e. $\lambda \in \Lambda$,

$$
\operatorname{deg}\left(u_{j}(\cdot, \lambda)\right) \rightarrow \operatorname{deg}(u(\cdot, \lambda)) .
$$

The conclusion follows by picking any $\lambda$ outside a countable union of sets of measure zero. The uniqueness of the limit implies the convergence of the full sequence.

Remark 3. The above argument extends to the case where $X$ and $Y$ need not have the same dimension, and degree is replaced by homotopy classes. More precisely we have

Theorem 2'. Assume that $u \in W^{s, p}(\Omega, Y)$ and that (9) holds with $n=\operatorname{dim} X$, then there is a homotopy class $\mathcal{C}$ in $C^{0}(X, Y)$ such that

$$
u(\cdot, \lambda) \in \mathcal{C} \quad \text { for a.e. } \lambda \in \Lambda \text {. }
$$

Proof. When $\Lambda=(0,1)$ we may invoke Lemma A.20 in [5] to assert that two continuous maps which are homotopic within VMO are also homotopic in $C^{0}(X, Y)$.

In the general case we denote by $\left(\mathcal{C}_{k}\right), k=1,2, \ldots$, the homotopy classes of $C^{0}(X, Y)$ (the connected components of $C^{0}(X, Y)$ are countable since $C^{0}(X, Y)$ is separable). For every $v \in C^{0}(X, Y)$ we set

$$
\operatorname{deg} v=k \quad \text { provided } v \in \mathcal{C}_{k}
$$


and the above argument remains unchanged.

We conclude with a similar question in the VMO framework. Let $X, Y$ and $\Lambda$ be as in Section 1 and let

$$
u \in \operatorname{VMO}(\Omega, Y)
$$

Open Problem: Is it true that for a.e. $\lambda \in \Lambda$

$$
u(\cdot, \lambda) \in \operatorname{VMO}(X, Y)
$$

and if so, is $\operatorname{deg}(u(\cdot, \lambda))$ constant a.e. in $\Lambda$ ?

This question is also related to a question of H. Amann and result in [6] (p.332-333).

\section{REFERENCES}

[1] R. A. Adams, Sobolev Spaces, Academic Press, 1975.

[2] $\quad$ F. Bethuel, The approximation problem for Sobolev mappings between manifolds, Acta Math. 167 (1991), 153-206.

[3] F. Bethuel and F. Demengel, Extensions for Sobolev mappings between manifolds, Calc. Var. 3 (1995), 475-491.

[4] A. Boutet de Monvel-Berthier, V. Georgescu and R. Purice, A boundary value problem related to the Ginzburg-Landau model, Comm. Math. Phys. 142 (1991), 1-23.

[5] H. Brezis and L. Nirenberg, Degree Theory and BMO, Part I: Compact Manifolds without Boundaries, Selecta Math. 1 (1995), 197-263.

[6] H. Brezis and L. Nirenberg, Degree Theory and BMO, Part II: Compact Manifolds with Boundaries, Selecta Math. 2 (1996), 1-60.

[7] S. Lang, Differentiable Manifolds, Springer, 1985.

[8] L. Nirenberg, Topics in Nonlinear Functional Analysis, Courant Institute Lecture Notes (1974).

[9] J. Rubinstein and P. Sternberg, Homotopy classification of minimizers of the Ginzburg-Landau energy and the existence of permanent currents, Comm. Math. Phys. 179 (1996), 257-263.

[10] H. Triebel, Interpolation Theory, Function Spaces, Differential Operators, North Holland, 1978.

(1) Institut Universitaire de France and Analyse Numerique, Université P. et M. Curie, 4 Pl. Jussieu, 75252 Paris Cedex 05, France;

(2) Department of Mathematics, Rutgers University, Piscataway, NJ 08854,USA;

(3) Departement de Mathematiques, université Paris-Sud, Bat. 425, 91405 Orsay Cedex, FRANCE;

(4) Courant Institute, New York University, 251 Mercer St., New York, NY 10012, USA 